\title{
Exciton Fine-Structure Splitting in Self- Assembled Lateral InAs/GaAs Quantum-Dot Molecular Structures
}

\author{
Stanislav Filippov, Yuttapoom Puttisong, Yuqing Huang, Irina A Buyanova, Suwaree \\ Suraprapapich, Charles. W. Tu and Weimin Chen
}

\section{Linköping University Post Print}

\section{Tweet}

N.B.: When citing this work, cite the original article.

Original Publication:

Stanislav Filippov, Yuttapoom Puttisong, Yuqing Huang, Irina A Buyanova, Suwaree Suraprapapich, Charles. W. Tu and Weimin Chen, Exciton Fine-Structure Splitting in SelfAssembled Lateral InAs/GaAs Quantum-Dot Molecular Structures, 2015, ACS Nano, (9), 6, 5741-5749.

http://dx.doi.org/10.1021/acsnano.5b01387

Copyright: American Chemical Society http://pubs.acs.org/

Postprint available at: Linköping University Electronic Press http://urn.kb.se/resolve?urn=urn:nbn:se:liu:diva-118007 


\title{
Exciton Fine-Structure Splitting in Self-Assembled Lateral InAs/GaAs
}

\section{Quantum-Dot Molecular Structures}

Stanislav Fillipov, ${ }^{1, \dagger}$ Yuttapoom Puttisong, ${ }^{1, \dagger}$ Yuqing Huang, ${ }^{1}$ Irina A. Buyanova, ${ }^{1}$ Suwaree Suraprapapich, ${ }^{2}$ Charles W. Tu ${ }^{2}$ and Weimin M. Chen ${ }^{1, *}$

${ }^{1}$ Department of Physics, Chemistry and Biology, Linköping University, S-581 83

Linköping, Sweden

${ }^{2}$ Department of Electrical and Computer Engineering, University of California, La Jolla, CA 92093, USA

\begin{abstract}
Fine-structure splitting (FSS) of excitons in semiconductor nanostructures is a key parameter that has significant implications in photon entanglement and polarization conversion between electron spins and photons, relevant to quantum information technology and spintronics. Here, we investigate exciton FSS in self-organized lateral InAs/GaAs quantum-dot molecular structures (QMSs) including laterally-aligned double quantum dots (DQDs), quantum-dot clusters (QCs) and quantum rings (QRs), by employing polarization-resolved micro-photoluminescence ( $\mu \mathrm{PL})$ spectroscopy. We find a clear trend in FSS between the studied QMSs depending on their geometric arrangements, from a large FSS in the DQDs to a smaller FSS in the QCs and QRs. This trend is accompanied by a corresponding difference in the optical polarization directions of the excitons between these QMSs, namely, the bright-exciton lines are linearly polarized preferably along or perpendicular to the [1ํㅣ crystallographic axis in the DQDs that also defines the alignment direction of the two constituting QDs, whereas in the QCs and QRs the polarization directions are
\end{abstract}


randomly oriented. We attribute the observed trend in the FSS to a significant reduction of the asymmetry in the lateral confinement potential of the excitons in the QRs and QCs as compared with the DQDs, as a result of a compensation between the effects of lateral shape anisotropy and piezoelectric field. Our work demonstrates that FSS strongly depends on the geometric arrangements of the QMSs, which effectively tune the degree of the compensation effects and are capable of reducing FSS even in a strained QD system to a limit similar to strain-free QDs. This approach provides a pathway in obtaining high-symmetry quantum emitters desirable for realizing photon entanglement and spintronic devices based on such nanostructures, utilizing an uninterrupted epitaxial growth procedure without special requirements for lattice-matched materials combinations, specific substrate orientations and nanolithography.

\section{ToC graphic}

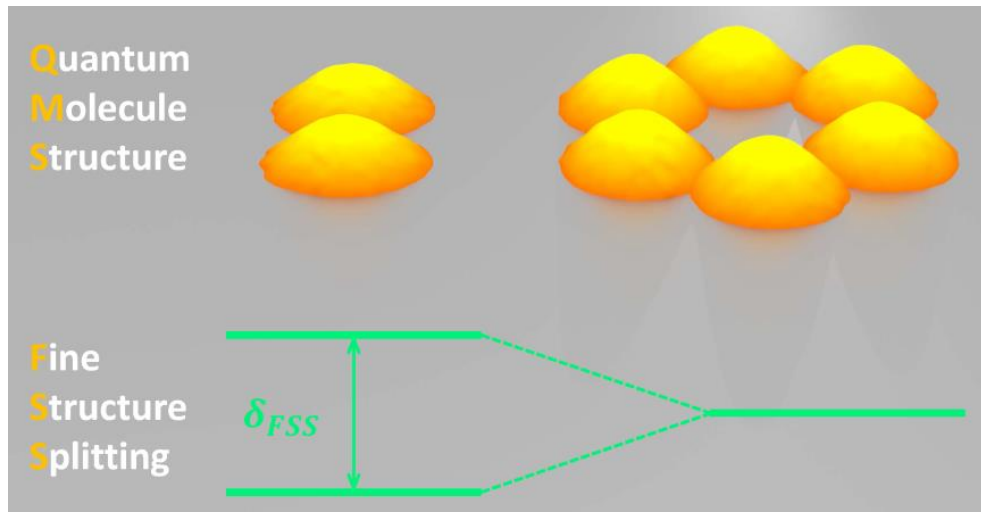

Keywords: InAs/GaAs, quantum dot, quantum-dot molecular structure, fine structure splitting, micro-photoluminescence, polarization 
Modern nanoscale engineering offers us a controllable way to build and design materials from nanoscale building-blocks. ${ }^{1-4}$ Among many interesting research topics in the field of nanoscience and nanotechnology, semiconductor quantum dots (QDs) and more complex quantum-dot molecular structures (QMSs) are important keys for realizing novel photonic, spintronic and quantum information devices including nanoscale solid-state lighting, ${ }^{5}$ entangled photon sources for quantum teleportation, quantum cryptography and distributed quantum computation, ${ }^{6-8}$ and spin light-emitting devices and quantum information processing utilizing electron spins. ${ }^{9-11}$ This is largely motivated by the expectations that QDs are efficient light emitters and possess long electron spin relaxation/coherence time. ${ }^{12,13}$ The photons emitted from the QDs are a result of excitonic recombination, which is associated with electron-heavy hole (e-hh) exchange-coupled pairs in most of the known semiconductor QD systems. It has now been well established that an anisotropic exchange interaction (AEI) can split the doublet of the bright neutral exciton states into two spinmixed, linearly polarized $|\mathrm{H}\rangle$ and $|\mathrm{V}\rangle$ exciton states, commonly referred to as a fine-structuresplitting (FSS). ${ }^{14}$ In highly strained self-assembled InAs/(Al)GaAs QDs, FSS has been shown to be in the range of $40-1000 \mu \mathrm{eV}^{15}$. This strong variation in FSS was suggested to be governed by effects of strain- and shape-induced wave function asymmetry of the excitons. ${ }^{15-16}$ The appearance of FSS becomes a major problem in many applications exploiting QDs. For example, FSS results in two distinguishable paths of exciton-biexciton cascade decays of different polarizations, inhibiting entanglement of the resulting photon pairs necessary for quantum information applications. Moreover, the presence of FSS signifies a reduced symmetry of the zero-dimensional quantum emitters where AEI induces strong electron spin relaxation and mixing of exciton spin states, undesirable for future applications in spintronics, spin-photonics and spin-based quantum information processing. During the past decade, we have witnessed intense research efforts and 
achievements in suppressing FSS by nanoscale engineering, e.g. droplet epitaxy to achieve lattice

matched QD systems, ${ }^{17}$ shape-controlled growth by nano-hole filling, ${ }^{18}$ and fabrication of QDs on a high-symmetry crystallographic plane $(111) \mathrm{A}^{19}$ or (111)B substrate. ${ }^{20}$

In this work, we demonstrate a different approach, namely geometrical engineering of selforganized QMSs, where FFS even in a strained QD system such as InAs/GaAs QDs can be significantly reduced to a limit similar to that achieved in strain-free QDs. This approach is attractive as the growth procedure is rather simple, using an uninterrupted molecular beam epitaxy (MBE) process within a single growth run, ${ }^{21,22}$ and does not require lattice-matching, special substrate orientations, nanolithography and site-controlled growth that could often be technically challenging and costly.

\section{Results/Discussion}

Typical atomic force microscopy (AFM) images of the studied QMSs are shown in Fig.1(a)-(d). First of all, all QMSs in a given sample are rather uniform in their overall sizes as a whole though the sizes of individual QDs forming each QMS noticeably differ. The QRs are slightly elongated along a $\langle 110\rangle$ crystallographic axis in the (001) plane. For the DQDs, the two dots constituting a DQD were preferably aligned along the [1ํㅣㄹ direction in the (001) plane, though each individual dot is slightly elongated along the orthogonal [110] direction, see Fig.2(d) for a close-up AFM image. Two samples of the DQDs, differing in QD sizes, are studied in this work to examine the size effect. In the QCs, each cluster typically consists of 5-7 dots that are rather symmetrically arranged around the center. There are, however, large variations in sizes and the directions of the elongation axes between individual QDs within each QC. 
The zero-dimensional nature of the studied QMSs was confirmed by the observation of discrete sharp excitonic emission lines, as revealed in our $\mu$ PL studies of individual QR, DQD and QC shown in Fig.1(e)-(h). The spectral linewidth of $\sim 15-35 \mu \mathrm{eV}$ is in agreement with previous studies of QMSs. ${ }^{23-25}$ We note that this linewidth is much larger than what is expected from the lifetime broadening or the spectral resolution of our instrument $(\sim 5 \mu \mathrm{eV})$. We believe that the main reason for the observed inhomogeneous broadening of the PL linewidth is the random fluctuations of local potentials as a result of $e . g$. fluctuating electric fields induced by charges at neighboring QDs and C-acceptors in the GaAs layers. ${ }^{26}$ By measuring $\mu \mathrm{PL}$ spectra in two orthogonal polarization directions, the doublet nature of the bright exciton ground states can be clearly resolved for each QMS. To determine the polarization axes of these two PL lines, we measured $\mu \mathrm{PL}$ spectra as a function of the rotation angle $\phi$ of the linear polarization analyzer with respect to the $[1 \overline{1} 0]$ crystallographic axis. Fig.2(a) displays representative results, from an individual DQD as an example, which show a strong angular dependence in the relative PL intensities of the two excitonic lines while their energy positions and thus the FSS remain constant for the given DQD as expected. A polar plot of the intensity of each PL line is shown in Fig.2(b), obtained from a deconvolution analysis of each $\mu \mathrm{PL}$ spectrum by two PL components of the fixed energies. The angular variation in the intensity of each PL line can be modeled by the following equation:

$$
I=I_{\min } \sin ^{2}(\phi-\theta)+I_{\max } \cos ^{2}(\phi-\theta),
$$

where $I_{\max }\left(I_{\min }\right)$ defines the maximum (minimum) PL intensity for a given PL line. The phase $\theta$ is the angle at which the concerned PL line reaches $I_{\max }$, which corresponds to the angle between the optical polarization axis and the [1ํㅣㄹ axis. An example of such simulations is given by the solid lines in Fig.2(b), where the lower-energy line is found to be polarized along the [1ํㅣㄹ direction whereas the higher-energy line is orthogonally polarized (i.e. along the [110] axis). This 
polarization behavior is characteristic for an AEI-induced FSS of the bright neutral exciton states. ${ }^{14}$ As illustrated in Fig.2(c), without AEI the two bright states of the neutral excitons are two-fold degenerate with the eigenstates of $|+1\rangle$ and $|-1\rangle$ that are $\sigma^{+}$and $\sigma^{-}$active, respectively. The electron spin is preserved in these hh exciton states, namely, electron spin-down in $|+1\rangle$ and electron spinup in $|-1\rangle$. The action of a non-zero AEI reduces the symmetry (for example from $\mathrm{D}_{2 \mathrm{~d}}$ to $\mathrm{C}_{2 \mathrm{v}}$ ), which lifts the degeneracy of the doublet and splits it into two non-degenerate exciton sublevels denoted by $\mathrm{H}$ and $\mathrm{V}$. Their eigenstates are $|\mathrm{H}\rangle=(|+1\rangle+|-1\rangle) / \sqrt{2}$ and $|\mathrm{V}\rangle=(|+1\rangle-|-1\rangle) / \sqrt{2}$ [14], respectively, in which the electron spin states are strongly mixed. These two sublevels give rise to two linearly and orthogonally polarized PL lines, i.e. $\sigma^{H}$ for $\mathrm{H}$ and $\sigma^{V}$ for $\mathrm{V}$. The magnitude of the FSS $\left(\delta_{F S S}\right)$ can be determined by the energy separation between the $\mathrm{H}$ and $\mathrm{V}$ sublevels, e.g. $\left|\delta_{F S S}\right|=\left|E_{H}-E_{V}\right| \approx 40 \mu \mathrm{eV}$ for the DQD-1 shown in Fig.2(a). The linear polarization directions of the $\mathrm{H}$ and $\mathrm{V}$ state are defined by the directions of exciton wave function asymmetry under the influence of a directional confining potential. In the case of the DQD shown in Fig.2(a) and 2(b), the lower-lying state $E_{V}$ is polarized along the [1ํㅣ axis whereas the higher-energy state $E_{H}$ is polarized along the [110] axis as indicated in Fig.2(d).

In order to obtain more detailed information on the average value of the FSS and the polarization axis of the exciton sublevels and to reliably compare them between the studied QMSs, we carried out a systematic and statistical study of the FSS in a large number of individual QMSs for each QMS ensemble. The measured distributions of the $\delta_{F S S}$ values and their absolute values $\left|\delta_{F S S}\right|$ in the studied QMSs are displayed in Fig.3(a)-(d) and Fig.3(e)-(h), respectively. The data points with $\left|\delta_{F S S}\right|<5 \mu \mathrm{eV}$ are not shown in Fig.3(a)-(h) because the corresponding single PL lines 
can be contributed by both neutral excitons with $\left|\delta_{F S S}\right|<5 \mu \mathrm{eV}$ (thus unresolved due to the spectral resolution of our $\mu$-PL system) and charged excitons such as positive and negative trions that remain spin degenerate due to the Kramers theorem. Since these two contributions cannot be definitely separated experimentally, no reliable data points for the concerned neutral excitons can be obtained. However, one would expect that such data points should fall within the statistical distributions given in Fig.3(a)-(h). Two prominent features can clearly be seen from the results shown in Fig.3(a)-(h). Firstly, the FSS in the DQDs is found to be noticeably larger than that obtained in the QRs and the QCs, both in $\delta_{F S S}$ and $\left|\delta_{F S S}\right|$ values as given in Table 1. Secondly, a sizable portion of the QRs and QCs appears to have a negative $\delta_{F S S}$ value whereas the corresponding proportion is significantly less in the DQDs. The positive (negative) sign of $\delta_{F S S}$ is assigned to a QMS when the lower-energy exciton line of the bright exciton doublet becomes more polarized along the $[1 \overline{1} 0]([110])$ axis. The situation of a negative $\delta_{F S S}$ arises when the polarization direction of the lower-lying exciton sublevel has undergone a rotation away from [1 10 ] by an angle $\theta$ larger than 45 degrees, such that its projected intensity along the [11̄0] axis becomes weaker than that along the [110] axis. The observed larger spread in the distribution of $\delta_{F S S}$ toward negative values is thus indicative of a strong deviation between individual QMSs within the ensemble in the optical polarization axis and thus the direction of the expansion of the exciton wave function.

This assertion is further supported by the statistical distribution in the angle $\theta$ between the polarization axis of the lower-energy exciton state and the [110] crystallographic axis, experimentally obtained from a detailed angular dependent study of the polarization with the aid 
of Eq.(1) in the same way as that shown in Fig.2(b). The results from the QMSs studied in this work are summarized in Fig.3(i)-(1). It is obvious that the polarization axis of the lower-energy exciton sublevel in the DQDs is largely pinned around $\theta=0$ degree. In other words, it is preferably aligned along the [1ํㅣㄹ axis. In sharp contrast, the polarization axis of the lower-energy exciton sublevel in the QRs and QCs is randomly oriented that signifies a lack of a preferred direction. This finding is consistent with the significantly larger spread in the sign of $\delta_{F S S}$ found in the QRs and QCs shown in Fig.3(a)-(d), as $\delta_{F S S}$ changes its sign when the optical polarization axis undergoes a large rotation by more than 45 degrees.

In principle, the observed FSS could originate either from the QMS as a whole or from individual QDs that make up the QMS. Judging from the different sizes of the individual QDs found within each DQD and QC from the AFM images shown in Fig.1, these individual QDs are highly improbable to have the same electronic energies for carriers and excitons. Therefore the excitons monitored under our experimental conditions, with low excitation density and at low measurement temperature, are most likely localized within a single QD. This is consistent with earlier studies of DQDs in which possible coupling between the two QDs in a DQD only occurs in the excited states of electrons. ${ }^{27,28}$ Consequently, the observed FSS of such a localized exciton is expected to be primarily determined by the anisotropy of the confinement potential in the QD where the exciton is localized. As the FSS induced purely by atomistic asymmetry is expected to be small $(<10 \mu \mathrm{eV}),{ }^{29}$ the observed FSS should be mainly caused by shape- and strain-induced wave function asymmetry. ${ }^{15-16}$ Previous theoretical studies $^{30}$ and recent $k \cdot p$ calculations with a configuration interaction ${ }^{31}$ have shown that the lower-lying bright exciton state should be polarized along the direction parallel to the structural elongation axis of the QD, when only the effect of 
shape-induced asymmetry is taken into account. Our experimental results show an opposite correlation in the DQDs, namely, the lower-lying bright exciton state is in fact polarized along the [110] axis that is perpendicular to the elongation axis (i.e [110]) of each of the two QDs constituting the DQD as shown in Fig.2(b) and 2(d). This finding thus rules out the effect of the shape-induced asymmetry of individual QDs as being the governing factor for the sizable FSS observed in the DQDs. This conclusion is further supported by our experimental results from the statistical distributions of the lateral aspect ratio of individual QDs in the studied DQDs, QRs and QCs, shown in Fig.4. Unlike the trend found for the FSS with the geometric arrangement of the QMSs, no correlation can be found between the aspect ratio and the FSS. Furthermore, the DQD1 sample with the smaller sizes and the larger lateral shape anisotropy of individual QDs than those in the DQD-2 sample should exhibit larger $\mathrm{FSS}^{15}$ if only the shape anisotropy is considered. This is inconsistent with our experimental finding that the FSS is in fact rather similar between the DQD-1 and DQD-2. These results show that factors other than the QD shape anisotropy must play a key role in determining the overall confinement potential of the excitons.

Previous studies in strain-driven self-assembled QDs have suggested the strain effect as a dominant factor for the observed strong $(>20 \mu \mathrm{eV})$ and directional FSS. ${ }^{15-16}$ If strain-induced piezoelectricity $(\mathrm{PZ})$ is included, the expansion of the exciton wave function (thus the direction of the polarization axis) can be either along the [110] or [1ํㅣ direction, depending on the relative importance of linear and quadratic terms of the PZ as well as the correlation between electron and hole wave functions which are pointed orthogonally to each other under the action of the PZ. ${ }^{32-34}$ The rotation of the polarization axis by 90 degrees away from the elongation direction of the QDs, as seen in the DQDs, provides evidence for an important role of a directional PZ field in inducing FSS. We therefore attribute the observed FSS to a combined effect of shape anisotropy and strain- 
induced piezoelectric field on the symmetry of the confinement potential of the excitons. These two effects compensate each other, and the resulting FSS thus depends on the degree of the compensation. In the case of the DQDs, the effect of the piezoelectric field substantially overcompensates the effect of the shape anisotropy such that the effective confinement potential is elongated along the $[1 \overline{1} 0]$ crystallographic axis despite that the physical shape of each QD of the pair is actually elongated along the [110] axis. This leads to a sizable FSS and the [1] 0$]$-oriented polarization axis of the lower lying bright exciton state. Since the degree of the shape anisotropy is similar among all studied QMSs, reflected by their similar lateral aspect ratios shown in Fig.4, a smaller FSS observed in the QRs and QCs as compared with that in the DQDs can then be explained by a reduction in the directional piezoelectric field so that its compensation to the effect induced by the shape anisotropy is more complete. The reduction in the piezoelectric field is related to a weak and less directional strain field experienced by each QR and each QD in the QCs, which is also responsible for the overall higher symmetry of these QMSs since their formation is strain driven. As the elongation direction of an individual QD in the QC is largely random and probably the piezoelectric field is auto-correlated with the QD shape anisotropy (thus strain field induced by In incorporation), the polarization axes of the bright exciton states also become randomly oriented in agreement with our experimental results. The suggested compensation between the effects induced by the shape anisotropy and the piezoelectric field is also consistent with our experimental finding that the FSS is nearly spectrally independent. This is because it has been shown that FSS induced by the same degree of the shape anisotropy should increase with increasing photon energy (i.e. for smaller QDs) whereas that the piezoelectric field does the opposite, ${ }^{15}$ resulting in a cancellation in the spectral dependence of the FSS between the two effects. As to possible sources for the remaining small and non-vanishing FSS, besides an incomplete compensation between the 
effects of the shape anisotropy and the piezoelectric field, intrinsic effects such as interfacial symmetry lowering and its enhancement by atomistic elasticity, ${ }^{35}$ atomic-scale alloy randomness ${ }^{36}$ and fluctuation of charged defects in the environment nearby the QMSs could also lead to a small value of the FFS ( $\left.\delta_{F S S}<10 \mu \mathrm{eV}\right)$ that occur even in strain-free QD systems.

Our suggested model of the combined effect of the shape- and strain-induced anisotropy of the exciton confinement potential could also provide a possible explanation for the large FSS often reported in isolated single QDs grown in the strain-driven Stranski-Krastanov (SK) mode, i.e. 40$1000 \mu \mathrm{eV} .{ }^{15}$ In the case of such isolated QDs, the shape elongation is usually found to be along the [1ํㅣㄹ crystallographic axis that coincides with the piezoelectric field. In sharp contrast to the compensation between the effects of the shape anisotropy and the piezoelectric field in our QMSs, these two effects are additive in the case of these isolated QDs. This leads to a large FSS reported in the isolated QDs, even larger than the DQDs studied in our work as one could expect.

The attractiveness and feasibility of the general idea in our approach of suppressing FSS by exploiting lateral QMSs is further bolstered by the success of QMS growth by a variety of growth methods besides the partial-capping-regrowth process employed in this work, such as the technique combining MBE and in situ etching, ${ }^{37-39}$ droplet epitaxy, ${ }^{40}$ partial capping and annealing, ${ }^{41,42}$ a hybrid growth approach utilizing both droplet homoepitaxy and SK growth on self-assembled nanomound templates, ${ }^{43}$ etc.

\section{Conclusions}


In summary, our work demonstrated a possible pathway for suppressing FSS by nanoscale engineering exploiting the self-organized QMSs. We directly tackled the problem of strain-induced FSS by exploring controlled epitaxial growth conditions that lead to the formation of selective QMSs with a significantly reduced directional strain effect, without involving complicated growth and patterning procedures. More specifically, we started with QDs that could be used as the seeds to fabricate the QRs of a rather uniform size. These QRs possess a rather weak FSS, represented by an average and most probable absolute values of $\left|\delta_{F S S}\right|^{a v} \sim 30 \mu \mathrm{eV}$ and $\left|\delta_{F S S}\right|^{p} \sim 10 \mu \mathrm{eV}$, respectively. This FSS can be either enhanced or suppressed depending on the choices of parameters during the subsequent growth, which transforms the QRs into the DQDs or the QCs. The formation of the DQDs by the strain-driven partial-capping-regrowth method resulted in a larger FSS with $\left|\delta_{F S S}\right|^{a v} \sim 43 \mu \mathrm{eV}$ and $\left|\delta_{F S S}\right|^{p} \sim 45 \mu \mathrm{eV}$. The partial-capping-regrowth of the QRs at a higher temperature, on the other hand, led to the formation of the QCs with a small FSS with $\left|\delta_{F S S}\right|^{a v} \sim 21 \mu \mathrm{eV}$ and $\left|\delta_{F S S}\right|^{p} \sim 10 \mu \mathrm{eV}$ - the smallest FSS in all studied QMSs. This small FSS is equivalent to the values found for strain-free QDs such as GaAs/AlGaAs QDs reported in the literature, but can now be achieved in a nominally strained QD system based on InAs/GaAs QDs in a self-organized fashion. The physical mechanism for the observed trend in the FSS among the QMSs is discussed in terms of the compensation between the effects of the shape anisotropy and the piezoelectric field on the resulting confinement potential of the monitored excitons localized within individual QDs constituting the QMSs. Our work has thus demonstrated an attractive alternative approach for producing high-symmetry quantum emitters via geometric engineering of QMSs, desirable for future applications of entangled photons and electron/exciton spins in quantum information processing/communications and spintronics/spin-photonics. 


\section{Methods/Experimental}

The studied samples are a set of self-organized InGaAs/GaAs QMSs grown in the SK mode by gas-source MBE on a (001) semi-insulating GaAs substrate. They include QRs, lateral-aligned DQDs and QCs, formed during the partial-capping-regrowth process of self-assembled InAs QDs under different growth conditions. The QRs were formed by partially capping of InAs QDs by 6 monolayers (MLs) of GaAs at a substrate temperature of $470{ }^{\circ} \mathrm{C}$ under As2 overpressure. By depositing an additional 0.6 ML of InAs on the QRs, InAs DQDs were formed. However, if the substrates were heated to $520{ }^{\circ} \mathrm{C}$ in the deposition step for the $0.6 \mathrm{ML}$ of InAs, the QRs were transformed to QCs. All the samples are nominally undoped. For AFM studies, another layer of the same QMSs was regrown on the surface of each QMS sample. A detailed description of the QMS samples and the growth procedures can be found in Ref. 21 and Ref .22. We note that GaAs capping could in principle lead to structural changes of buried QMSs, as compared with the QMSs on the surface imaged by AFM. The extent of the changes is expected to critically depend on details in methods, procedures and conditions used during the growth, which affect the surface chemical potential, the migration length and anisotropy of In adatoms, and In-Ga intermixing. ${ }^{27,42,44,45}$ It is extremely difficult to experimentally access and determine the exact shapes of the buried QMSs, however, as any invasive approach can alter QMS structures by itself. AFM is at the moment the best method available for the purpose and has been used in nearly all studies reported in the literature and also in our work. Considering the relatively low temperatures and the arsenic species of $\mathrm{As}_{2}$ used during the growth of our QMSs, of which both should lead to a shorter In migration length, we believe that the overall lateral arrangements of the buried QMSs are expected to be similar to their counterparts on the surface imaged by AFM. 
The FSS of individual QMSs was studied by means of polarization-resolved $\mu \mathrm{PL}$ spectroscopy performed at $5 \mathrm{~K} . \mu \mathrm{PL}$ spectra were measured with a confocal system (Horiba-Jobin HR800) in the backscattering geometry. The continue-wave excitation source was either a wavelength-tunable Ti-sapphire laser (700-1000 $\mathrm{nm})$ or fixed-wavelength solid-state semiconductor diode lasers, providing non-resonant optical excitation of the QMSs with photon energies above the GaAs bandgap. The excitation spot was $\sim 1 \mu \mathrm{m}$ in diameter. The optical excitation power was kept low to avoid contributions from excited states and multi-exciton complexes. The PL signal was dispersed by a high-resolution spectrometer, with a spectral resolution of about $5 \mu \mathrm{eV}$, and detected by a charge-coupled-device (CCD) detector. For optical polarization studies, a linear-polarization analyzer, consisting of a rotatable half-wave-plate in conjunction with a fixed linear polarizer, was placed in the detection path before the entrance slit of the spectrometer. Due to weak PL signals, a long accumulation time on the order of ms was employed for acquisition of each PL spectrum. This is about $10^{6}$ times of the exciton lifetime, and as a result the measured PL spectrum was a statistical average over a large number of exciton decay events during which the monitored QD experienced significant local potential fluctuations. We should note that the potential fluctuations induce the PL line broadening mainly through a slight shift of the exciton energy. FSS measured from the PL peak positions, corresponding to the most probable local potential, is expected to be largely unaffected. Therefore the impact of the PL line broadening on our analysis of FSS in the QMSs can be neglected.

\section{AUTHOR INFORMATION}

\section{Corresponding Author:}


*E-mailwmc@ifm.liu.se (W.M.C.).

\section{Notes}

${ }^{\dagger}$ F.S. and Y.P contributed equally to this work.

The authors declare no competing financial interest.

\section{ACKNOLEDGMENTS}

This work was supported by Linköping University through the Professor Contracts, the Swedish Research Council (Grant No. 621-2011-4254), and the Linköping Linnaeus Initiative for Novel Functional Materials (LiLI-NFM) supported by the Swedish Research Council (contract number 2008-6582). We also appreciate a fruitful discussion about the FSS with Dr. Fredrik Karlsson. 


\section{REFERENCES}

1 Kroutvar, M.; Ducommun, Y.; Heiss, D.; Bichler, M.; Schuh, D.; Abstreiter, G.; Finley, J. J. Optically Programmable Electron Spin Memory Using Semiconductor Quantum Dots. Nature 2004, 432, 81-84.

2 Holub, M.; Bhattacharya, P. Spin-Polarized Light-Emitting Diodes and Lasers. J. Phys. D. Appl. Phys. 2007, 40, R179-R203.

3 Awschalom, D. D.; Loss, D.; Samarth, N. Semiconductor Spintronics and Quantum Computation (Springer, 2002)

4 Loss, D.; Divincenzo, D. P. Quantum Computation with Quantum Dots. Phys. Rev. A. 1998, 57, 120-126.

5 Knill, E.; Laflamme, R.; Milburn, G. J. A Scheme for Efficient Quantum Computation with Linear Optics. Nature 2001, 409, 46-52.

6 Gisin, N.; Ribordy, G.; Tittel, W.; Zbinden, H . Quantum Cryptography. Rev. Mod. Phys. 2002, 74, 145-195.

7 De Rinaldis, S.; D’Amico, I.; Rossi, F. Intrinsic Electric Field Effects on Few-Particle Interactions in Coupled GaN Quantum Dots. Phys. Rev. B, 2004, 69, 235316.

8 Bouwmeester, D.; Ekert, A.; Zeilinger, A. The Physics of Quantum Information (Springer, 2000).

9 Imamoglu, A.; Awschalom, D. D.; Burkard, G.; DiVincenzo, D. P.; Loss, D.; Sherwin, M.; Small, A. Quantum Information Processing Using Quantum Dot Spins and Cavity QED. Phys. Rev. Lett. 1999, 83, 4204.

10 Koppens, F. H. L.; Buizert, C.; Tielrooij, K. J.; Vink, I. T.; Nowack, K. C.; Meunier, T.; Kouwenhoven, L. P.; Vandersypen, L. M. K. Driven Coherent Oscillations of a Single Electron Spin in a Quantum dot. Nature 2006, 442, 766-771.

11 Petta, J. R.; Johnson, A. C.; Taylor, J. M.; Laird, E. A.; Yacoby, A.; Lukin, M. D.; Marcus, C. M.; Hanson, M. P.; Gossard, A. C. Coherent Manipulation of Coupled Electron Spins in Semiconductor Quantum Dots. Science 2009, 309, 2180-2184.

12 Elzerman, J. M.; Hanson, R.; Willems van Beveren, L. H.; Witkamp, B.; Vandersypen, L. M. K.; Kouwenhoven, L. P. Single-Shot Read-Out of an Individual Electron Spin in a Quantum Dot. Nature 2004, 430, 431-435.

13 Oulton, R.; Greilich, A.; Verbin, S.; Cherbunin, R.; Auer, T.; Yakovlev, D.; Bayer, M.; Merkulov, I.; Stavarache, V.; Reuter, D. et al. Subsecond Spin Relaxation Times in 
Quantum Dots at Zero Applied Magnetic Field Due to a Strong Electron-Nuclear Interaction. Phys. Rev. Lett. 2007, 98, 107401.

14 Bayer, M.; Ortner, G.; Stern, O.; Kuther, A.; Gorbunov, A.A.; Forchel, A.; Hawrylak, P.; Fafard, S.; Hinzer, K.; Reinecke, T.L. et al. Fine Structure of Neutral and Charged Excitons in Self-assembled $\mathrm{In}(\mathrm{Ga}) \mathrm{As} /(\mathrm{Al}) \mathrm{GaAs}$ Quantum Dots. Phys. Rev. B 2002, 65, 195315.

15 Seguin, R.; Schliwa, A.; Rodt, S.; Pötschke, K.; Pohl, U.; Bimberg, D. Size-Dependent Fine-Structure Splitting in Self-Organized InAs/GaAs Quantum Dots. Phys. Rev. Lett. 2005, 95, 257402.

16 Young, R. J.; Stevenson, R. M.; Shields, A. J.; Atkinson, P.; Cooper, K.; Ritchie, D. A.; Groom, K. M.; Tartakovskii, A.; I. Skolnick, M. S. Inversion of Exciton Level Splitting in Quantum Dots. Phys. Rev. B 2005, 72, 113305.

17 Abbarchi, M.; Mastrandrea, C.; Kuroda, T.; Mano, T.; Sakoda, K.; Koguchi, N.; Sanguinetti, S.; Vinattieri, A.; Gurioli, M. Exciton Fine Structure in Strain-Free GaAs/Al ${ }_{0.3} \mathrm{Ga}_{0.7} \mathrm{As}$ Quantum Dots: Extrinsic Effects. Phys. Rev. B 2008, 78, 125321.

18 Huo, Y. H.; Rastelli, A.; Schmidt, O. G. Ultra-small Excitonic Fine Structure Splitting in Highly Symmetric Quantum Dots on GaAs (001) Substrate. Appl. Phys. Lett. 2013, 102, 152105.

19 Kuroda, T.; Mano, T.; Ha, N.; Nakajima, H.; Kumano, H.; Urbaszek, B.; Jo, M.; Abbarchi, M.; Sakuma, Y.; Sakoda, K.; Suemune, I.; Marie, X. et al. Symmetric Quantum Dots as Efficient Sources of Highly Entangled Photons: Violation of Bell's Inequality Without Spectral and Temporal Filtering. Phys. Rev. B 2013, 88, 041306(R).

20 Mohan, A.; Felici, M.; Gallo, P.; Dwir, B.; Rudra, A.; Faist, J.; Kapon, E. Retracted Polarization-Entangled Photons Produced with High-Symmetry Site-Controlled Quantum Dots. Nat. Photonics 2010, 4, 302-306.

21 Suraprapapich, S.; Panyakeow, S.; Tu, C. W. Effect of Arsenic Species on the Formation of (Ga)InAs Nanostructures After Partial Capping and Regrowth. Appl. Phys. Lett. 2007, 90, 183112.

22 Suraprapapich, S.; Shen, Y. M.; Odnoblyudov, V. A.; Fainman, Y.; Panyakeow, S.; Tu, C. W. Self-assembled lateral Bi-quantum-dot molecule formation by gas-source molecular beam epitaxy. J. Cryst. Growth 2007, 301, 735-739.

23 Warburton, R. J.; Schaflein, C.; Haft, D.; Bickel, F.; Lorke, A.; Karrai, K.; Garcia, J. M.; Schoenfeld, W.; Petroff, P. M. Optical Emission From a Charge-Tunable Quantum Ring. Nature 2000, 405 926-929. 
24 Muñoz-Matutano, G.; Royo, M.; Climente, J. I.; Canet-Ferrer, J.; Fuster, D.; AlonsoGonzález, P.; Fernández-Martínez, I.; Martínez-Pastor, J.; González, Y. et al. Charge Control in Laterally Coupled Double Quantum Dots. Phys. Rev. B 2011, 84, 041308.

25 Creasey, M.; Lee, J.; Wang, Z.; Salamo, G. J.; Li, X. Self-Assembled InGaAs Quantum Dot Clusters with Controlled Spatial and Spectral Properties. Nano Lett. 2012, 12, 5169 5174.

26 Zhou, X. R.; Lee, J. H.; Salamo, G. J.; Royo, M.; Climente, J. I.; Doty, M. F. Coulomb Interaction Signatures in Self-Assembled Lateral Quantum Dot Molecules. Phys. Rev. B 2013, 87, 125309.

27 Zhou, X.; Sanwlani, S.; Liu, W.; Lee, J. H.; Wang, Z. M.; Salamo, G.; Doty M. F. Spectroscopic Signatures of Many-Body Interactions and Delocalized States in SelfAssembled Lateral Quantum Dot Molecules. Phys. Rev. B 2011, 84, 205411.

28 Heldmaier, M.; Seible, M.; Hermannstadter, C.; Witzany, M.; Roübach, R.; Jetter, M.; Michler, P.; Wang, L.; Rastelli, A.; Schmidt, O. G. Excited-State Spectroscopy of Single Lateral Self-Assembled InGaAs Quantum Dot molecules. Phys. Rev. B 2012, 85, 115304.

29 Bester, G.; Nair, S.; Zunger A. Pseudopotential Calculation of the Excitonic Fine Structure of Million-Atom Self-Assembled $\mathrm{In}_{1_{-} \mathrm{x}} \mathrm{Ga}_{\mathrm{x}} \mathrm{As} / \mathrm{GaAs}$ Quantum Dots. Phys. Rev. B. 2003, 67, 161306(R).

30 Takagahara, T. Theory of Exciton Doublet Structures and Polarization Relaxation in Single Quantum Dots. Phys. Rev. B 2000, 62, 16840-16855.

31 Tong, H.; Wu, M. W. Theory of Excitons in Cubic III-V Semiconductor GaAs, InAs and GaN Quantum Dots: Fine Structure and Spin Relaxation. Phys. Rev. B 2011, 83, 235323.

32 Bester, G.; Zunger, A.; Wu, X.; Vanderbilt, D. Effects of Linear and Nonlinear Piezoelectricity on the Electronic Properties of InAs/GaAs Quantum Dots. Phys. Rev. B 2006, 74, 081305.

33 Schliwa, A.; Winkelnkemper, M.; Bimberg, D. Impact of Size, Shape, and Composition on Piezoelectric Effects and Electronic Properties of In(Ga)As/GaAs Quantum Dots. Phys. Rev. B 2007, 76, 205324.

34 Stier, O.; Grundmann, M.; Bimberg, D. Electronic and Optical Properties of Strained Quantum Dots Modeled by 8-Band k·p Theory. Phys. Rev. B 1999, 59, 5688-5701.

35 Bester, G.; Zunger, A. Cylindrically Shaped Zinc-Blende Semiconductor Quantum Dots Do Not Have Cylindrical Symmetry: Atomistic Symmetry, Atomic Relaxation, and Piezoelectric Effects. Phys. Rev. B 2005, 71, 045318. 
36 Mlinar, V.; Zunger, A. Effect of Atomic-Scale Randomness on the Optical Polarization of Semiconductor Quantum Dots. Phys. Rev. B 2009, 79, 115416.

37 Hermannstadter, C.; Witzany, M.; Beirne, G. J.; Schulz, W. M.; Eichfelder, M.; Rossbach, R.; Jetter, M.; Michler, P.; Wang, L.; Rastelli, A.; Schmidt, O. G. Polarization Fine Structure and Enhanced Single-Photon Emission of Self-Assembled Lateral InGaAs Quantum Dot Molecules Embedded in a Planar Microcavity. J. Appl. Phys. 2009, 105, 122408.

38 Peng, J.; Hermannstadter, C.; Witzany, M.; Heldmaier, M.; Wang, L.; Kiravittaya, S.; Rastelli, A.; Schmidt O. G.; Michler, P.; Bester, G. Heterogeneous Confinement in Laterally Coupled InGaAs/GaAs Quantum Dot Molecules Under Lateral Electric Fields. Phys. Rev. B 2010, 81, 205315.

39 Songmuang, R.; Kiravittaya, S.; Schmidt, O. G. Formation of Lateral Quantum Dot Molecules Around Self-Assembled Nanoholes. Appl. Phys. Lett. 2003, 82, 2982.

40 Mano, T.; Koguchi, N. Nanometer-Scale GaAs Ring Structure Grown by Droplet Epitaxy. J. Cryst. Growth 2005, 278, 108-112.

41 Offermans, P.; Koenraad, P. M.; Wolter J. H.; Granados, D.; Garcia J. M.; Fomin, V. M.; Gladilin, V. N.; Devreese, J. T. Atomic-Scale Structure of Self-Assemble In(Ga)As Quantum Rings in GaAs. Appl. Phys. Lett. 2005, 87, 131902.

42 Wang, L.; Rastelli, A.; Schmidt, O. G. Structural and Optical Properties of In (Ga) As/ GaAs Quantum Dots Treated by Partial Capping and Annealing. J. Appl. Phys. 2006, 100, 064313.

43 Lee, J. H.; Wang, Zh. M.; Strom, N. W.; Mazur Yu. I.; Salamo, G. J. InGaAs Quantum Dot Molecules Around Self-Assembled GaAs Nanomound Template. Appl. Phys. Lett. 2006, 89, 202101.

44 Songmuang, R.; Kiravittaya, S.; Schmidt, O. G. Shape Evolution of InAs Quantum Dots During Overgrowth. J. Cryst. Growth 2003, 249, 416-421.

45 Lee, J. H.; Wang, Z. M.; Dorogan, V. G.; Mazur, Y. I.; Salamo G. J. Evolution of Various Nanostructures and Preservation of Self-Assembled InAs Quantum Dots During GaAs Capping. IEEE Transactions on Nanotechnology 2010, 9, 149-156. 
Table 1. The average and most probable values of $\delta_{F S S}$ and $\left|\delta_{F S S}\right|$ determined from the statistical distributions in the studied QMS ensembles. The average values are calculated by $\delta_{F S S}^{a v}=\frac{\sum_{i} n_{i} \delta_{F S S}^{i}}{\sum_{i} n_{i}}$ and $\left|\delta_{F S S}\right|^{a v}=\frac{\sum_{i} m_{i}\left|\delta_{F S S}^{i}\right|}{\sum_{i} m_{i}}$, respectively, where $n_{i}\left(m_{i}\right)$ represents the number of the QMS with the FSS value of $\delta_{F S S}^{i}\left(\left|\delta_{F S S}^{i}\right|\right)$. The most probable values of $\delta_{F S S}^{p}$ and $\left|\delta_{F S S}\right|^{p}$ correspond to the peak positions in the Gaussian distributions shown in Fig.3(a)-(d) and in the experimental data in Fig.3(e)-(h), respectively. The predicted values around zero FSS from the Gaussian distribution of $\delta_{F S S}$ are used to estimate the values given in the table.

\begin{tabular}{|c|c|c|c|c|}
\hline & QR & DQD-1 & DQD-2 & QC \\
\hline Average $\delta_{F S S}^{a v}$ & 12 & 38 & 28 & 7 \\
\hline Most probable $\delta_{F S S}^{p}$ & 16 & 42 & 39 & 7 \\
\hline Average $\left|\delta_{F S S}\right|^{a v}$ & 28 & 43 & 43 & 19 \\
\hline Most probable $\left|\delta_{F S S}\right|^{p}$ & 10 & 50 & 40 & 10 \\
\hline
\end{tabular}



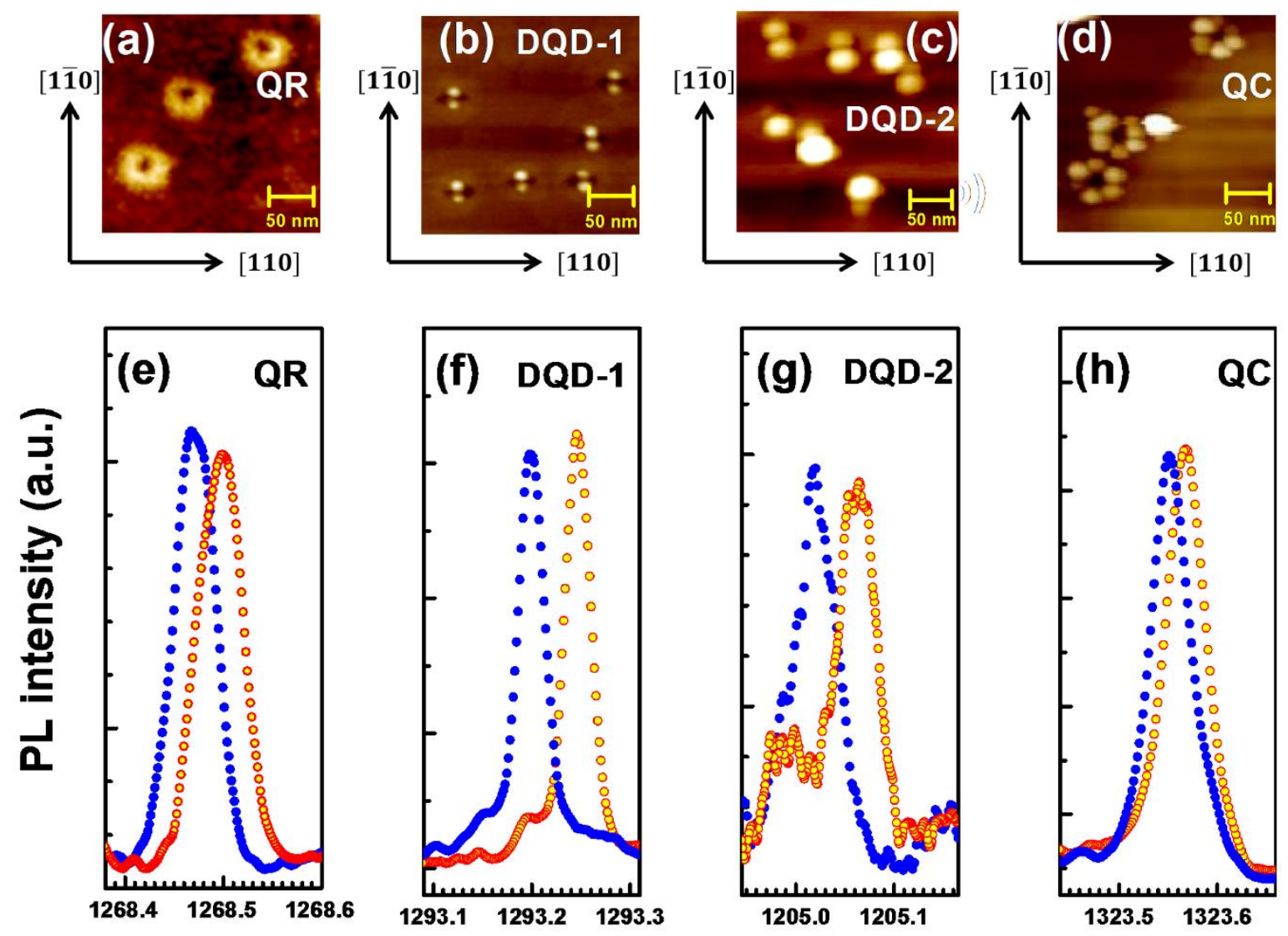

Emission energy (meV)

Figure 1 (a)-(d) AFM images of the studied QRs, laterally aligned DQDs and QCs. (e)-(h) Representation $\mu \mathrm{PL}$ spectra obtained from single $\mathrm{QR}, \mathrm{DQD}$ and $\mathrm{QC}$, when the axis of the linear polarization analyzer was aligned along the [11̄0] (the solid symbols) and [110] (the open symbols) axes. The displayed spectra from the QRs and QCs are special cases when the polarization axes of the exciton states coincide with these crystallographic axes. 

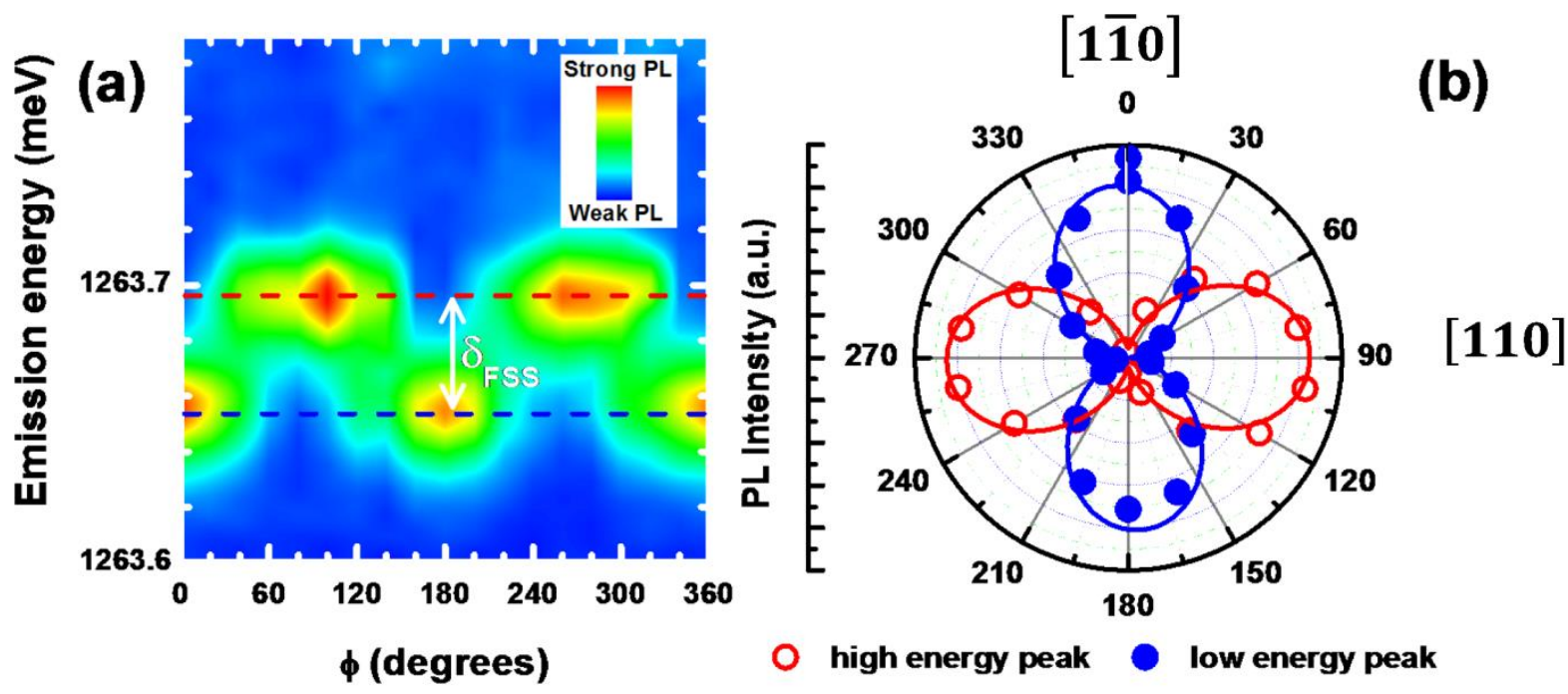

(C) high symmetry low symmetry
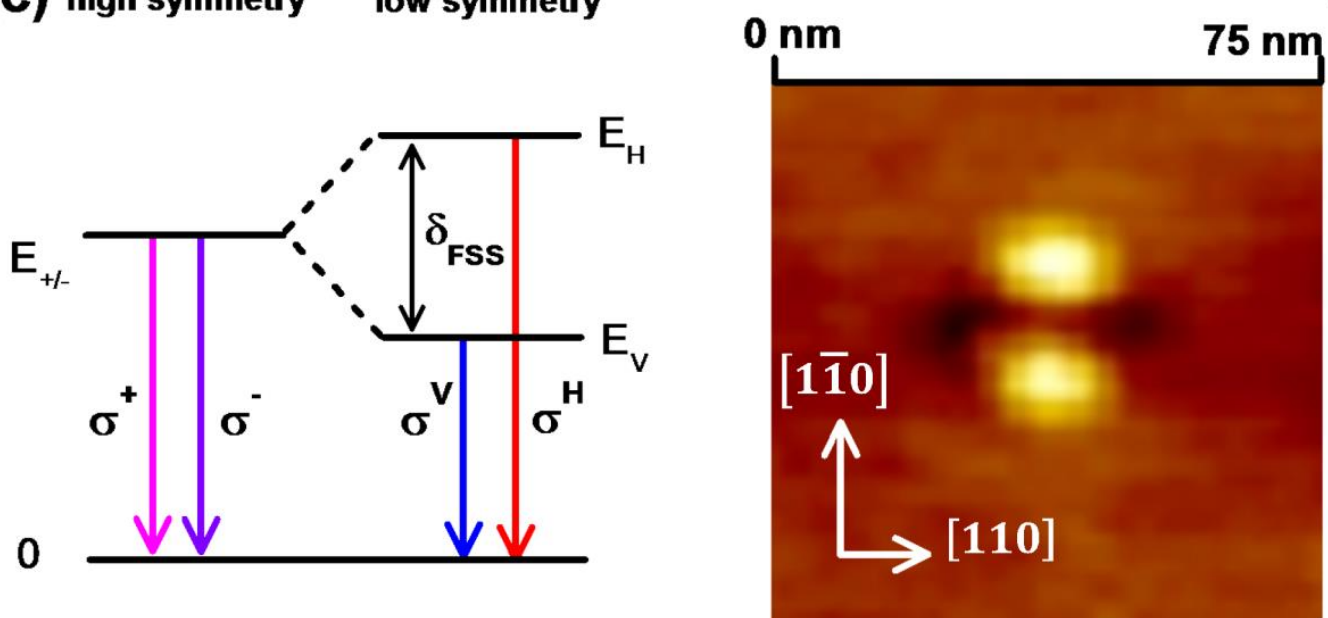

Figure 2 (a) 2D mapping of the bright ground states of the neutral exciton. (b) A polar plot of the integrated intensity of the lower-energy (the filled circles) and higher-energy (the open circles) exciton states. The solid lines are the simulated curves by Eq.(1). (c) Energy diagram representing the effect of AEI on the bright exciton states. (d) A close-up AFM image of a single DQD. All data were obtained from an individual DQD of the DQD-1 sample. 

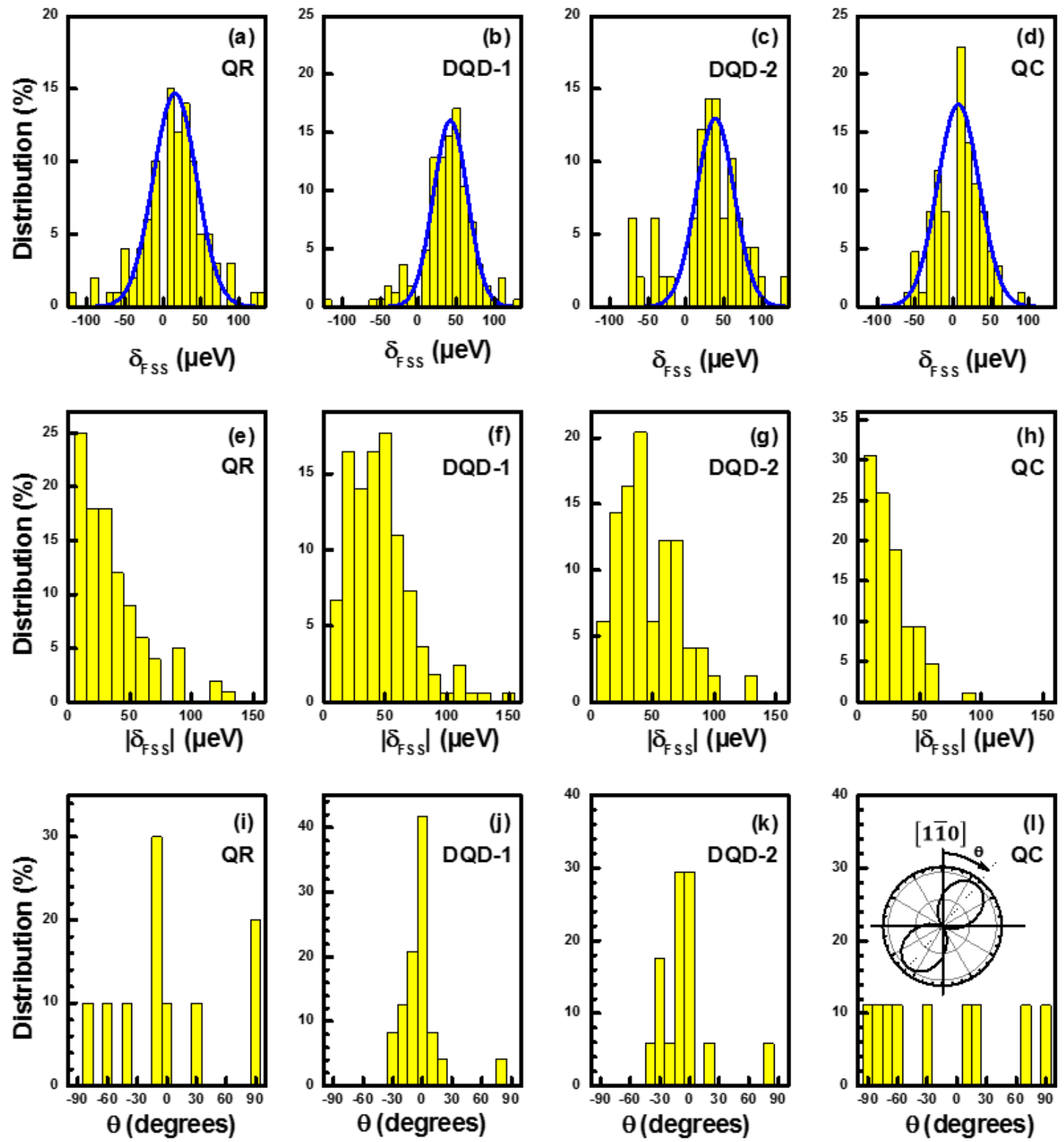

Figure 3 Statistical distributions of the $\delta_{F S S}$ (a-d) and their absolute values $\left|\delta_{F S S}\right|$ (e-h) obtained from the studied QMSs. The solid curves in a-d represent a Gaussian distribution function, as a guide to the eye. (i)-(l) Angular distributions of the polarization axis of the lower-lying exciton state from the studied QMSs, with respective to the [11̄0] axis as defined by the sketch in (l). The total numbers of the individual QMSs used in the statistical distributions are 100 in (a) and (e), 164 in (b) and (f), 49 in (c) and (g), 85 in (d) and (h), 10 in (i), 24 in (j), 17 in (k) and 9 in (l). 

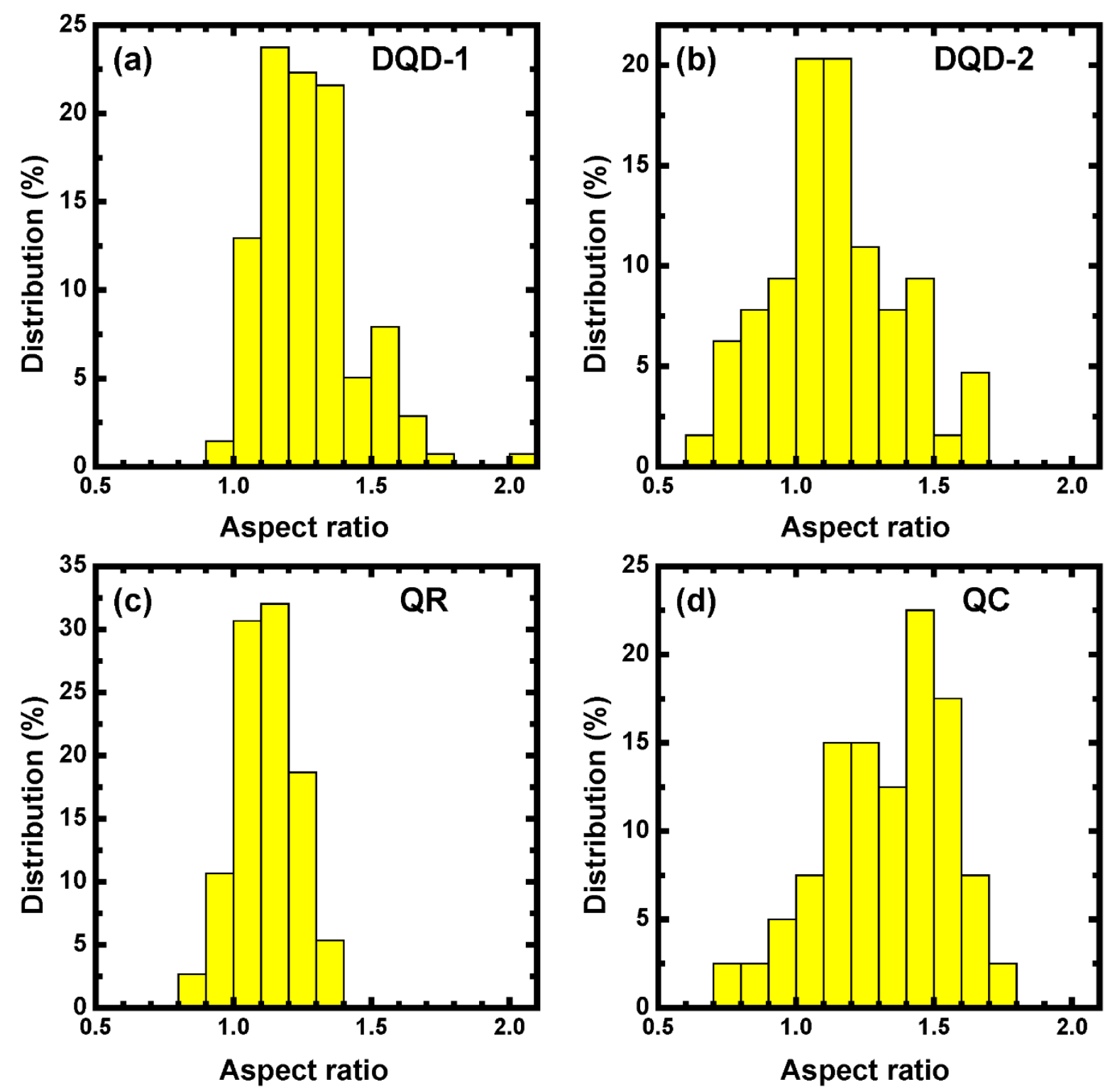

Figure 4 Statistical distributions of the lateral aspect ratios (long axis length/short axis length) obtained from the studied QMSs. 